\title{
HCV genotype 5: Epidemiology and spread of an uncommon genotype
}

\author{
Jannick Verbeeck, ${ }^{a}$, Hélène Peigue-Lafeuilleb, ${ }^{c}$, R. Stefan Ross ${ }^{\mathrm{d},}$ Armand Abergel ${ }^{\mathrm{e}}$, Frederik Nevens ${ }^{\mathrm{f}}$, Schalk Van der \\ Merwe $^{g}$, Marc Van Ranst ${ }^{\mathrm{a}}$, and Cécile Henquell ${ }^{\mathrm{c}}$ \\ a Laboratory of Clinical and Epidemiological Virology, Rega Institute for Medical Research, University of Leuven, Leuven, \\ Belgium \\ ${ }^{\mathrm{b}}$ Laboratory of Virology-EA 3843, Faculty of Medicine, Auvergne University, Clermont-Ferrand, France \\ ${ }^{c}$ Laboratory of Virology, Gabriel Montpied Hospital, CHU of Clermont-Ferrand, France \\ ${ }^{d}$ Institute of Virology, National Reference Center for Hepatitis C, Essen University Hospital, University of Duisburg-Essen, \\ Essen, Germany \\ e Department of Gastro-enterology, Hôtel-Dieu, CHU of Clermont-Ferrand, France \\ fDivision of Hepatology, University Hospital Gasthuisberg, Leuven, Belgium \\ ${ }^{\mathrm{g}}$ Hepatology/GI-research laboratory, Institute of Pathology, Basic Medical Sciences, University of Pretoria, South Africa
}

Hepatitis $\mathrm{C}$ virus (HCV) is a leading cause of progressive liver disease, including chronic hepatitis, cirrhosis and hepatocellular carcinoma. As is the case for other RNA viruses, HCV viral diversity involves important clinical implications. HCV isolates from Western Europe and North America have limited sequence diversity, resulting from the recent introduction of a few strains, such as subtypes $1 \mathrm{a}, 1 \mathrm{~b}$ or $3 \mathrm{a}$, from endemic areas (Simmonds et al., 2005). These 'epidemic' strains have spread rapidly in the 20th century via blood transfusion, use of infected blood products, hemodialysis and intravenous drug use, and are characterized by a high prevalence and a global distribution. Since they are mostly prevalent in developed and rich countries, the majority of epidemiological studies focus on these genotypes. Circulation patterns of HCV genotypes in non-western countries are less documented. However, a large genomic diversity is found for HCV genotypes 1, 2 and 4 in Africa, and for genotypes 3 and 6 in India and Southeast Asia, suggesting a long-term infection in these areas (Pybus et al., 2001).

In contrast to other HCV genotypes, there is an evident lack of data on the epidemiological history of HCV genotype 5. For many years, this uncommon HCV genotype was believed to be confined to the northern part of South Africa and was considered as anecdotal elsewhere. However, the epidemiology of this HCV genotype seems to be more diverse than first thought. After a few reports on sporadic HCV genotype 5 infections in the United Kingdom, the Netherlands, Ireland, Australia, Canada, Brazil, and Germany ([Davidson et al., 1995], [Levi et al., 2002], [Murphy et al., 1994] and [Ross et al., 2000]), recent studies demonstrate that pockets of HCV genotype 5 infections can be found worldwide. Three European studies report an unusually high and local prevalence of HCV genotype 5 in Spain (Jover et al., 2001), in Belgium (Verbeeck et al., 2006) and in France (Henquell et al., 2004). Interestingly, these three studies show a high and very local prevalence ranging from $10.3 \%$ to $27.7 \%$ in areas characterized by close-knit societies; a higher mean age associated with HCV genotype 5 infections compared to other HCV genotype infections and transmission routes related to blood transfusion and hemodialysis, or unknown transmission. The cause of these isolated pockets of infection within these areas remains to be resolved. Interestingly, none of the patients have a known history of intravenous drug use. Phylogenetic analysis of the E1-E2 region of the French and Belgian strains (see Verbeeck et al., 2006 for the methods used to sequence all strains) revealed closely related sequences from five husband and wife couples, indicating that sexual or intrafamilial transmission is possibly underestimated. Limited data are available on the biological and clinicopathological features associated with HCV genotype 5 infections. Since large-scale clinical trials do not include a sufficient number of patients, the optimal treatment schedule remains unknown. For this reason, patients infected with HCV 
genotype 5 are treated with the same regimen as individuals infected with HCV genotype 1. Two recent retrospective French studies suggested that treatment response of HCV genotype 5 is better compared to HCV genotype 1 response, and is very similar to HCV genotypes 2 and 3 response ([Bonny et al., 2003] and [Legrand-Abravanel et al., 2004]). Substained virological response (SVR) is obtained in more than $60 \%$ of HCV genotype 5 patients treated with the combination of interferon (standard or pegylated) plus ribavirin for 48 weeks.

In order to extend our knowledge on this specific HCV genotype, further attempts have to be made to collect more epidemiological data from infected patients and samples from different geographical regions. Since HCV genotypes are important epidemiological markers and useful tools for investigating the spread $\mathrm{HCV}$, standard phylogenetic analysis combined with population genetic methods can be applied to estimate the epidemiological history from viral gene sequences and to identify the geographical origin of HCV genotype 5. Moreover, the different HCV genotypes are predictors of treatment response and have major implications on HCV vaccine development. Newly selected inhibitors of HCV replication, such as antipolymerases and anti-proteases, are optimized for HCV genotype 1 because of its higher prevalence in Europe, Japan and United States and its lower response rate to therapy. However, natural polymorphisms of viral targets are less known, or even unknown, for uncommon HCV genotypes, and may therefore be underestimated.

From the perspective of a virologist, any genotype-dependant difference in the efficiency of assays to accurately detect, quantify or genotype HCV, has to be carefully considered since clinical decisions are based upon the obtained results. For patients with chronic hepatitis $\mathrm{C}$ and for physicians who have to treat their patients, all HCV genotypes are of equal importance and optimal treatment schedules have to be determined for each HCV genotype. In this point of view, the less frequent genotypes deserve the same attention as the more common genotypes.

\section{References}

Bonny et al., 2003 C. Bonny, C. Roche, K. Randl, S. Ughetto, C. Henquell and N. Martineau et al., Treatment of interferon-naïve patients with HCV genotype 5 with interferon (or peginterferon) plus ribavirine results in a very high sustained viral response, Hepatology 38 (2003), p. A75.

Davidson et al., 1995 F. Davidson, P. Simmonds, J.C. Ferguson, L.M. Jarvis, B.C. Dow and E.A.C. Follett et al., Survey of major genotypes and subtypes of hepatitis $C$ virus using RFLP of sequences amplified from the 5' noncoding region, J Gen Virol 76 (1995), pp. 1197-1204.

Henquell et al., 2004 C. Henquell, C. Cartau, A. Abergel, H. Lauricesse, C. Regagnon and C. De Champs et al., High prevalence of hepatitis C virus type 5 in Central France evidenced by a prospective study from 1996 to 2002, J Clin Microbiol 42 (2004), pp. 3030-3035.

Jover et al., 2001 R. Jover, J. Pérez-Serra, F. de Vera, J.M. Alamo, C. Munoz and C. Yago et al., Infection by genotype 5a of HCV in a district of Southeast Spain, Am J Gastroenterol 96 (2001), p. 3042.

Legrand-Abravanel et al., 2004 F. Legrand-Abravanel, K. Sandrès-Saune, K. Barange, L. Alric, J. Moreau and P. Desmorat et al., Hepatitis $C$ virus genotype 5: epidemiological characteristics and sensitivity to combination therapy with interferon- $\alpha$ plus ribavirine, J Infect Dis 189 (2004), pp. 1397-1400.

J.E. Levi, D.T. Takaoka, R.H. Garrini, R.M. Fachini, R. Focaccia and E. de Bortholi Santos et al., Three cases of infection with hepatitis C virus genotype 5 among Brazilian hepatitis patients, J Clin Microbiol 40 (2002), pp. 2645-2647.

Murphy et al., 1994 D. Murphy, B. Willems and G. Delage, Use of the 5' noncoding region for genotyping hepatitis C virus, J Infect Dis 169 (1994), pp. 473-475. 
Pybus et al., 2001 O.G. Pybus, M.A. Charleston, S. Gupta, A. Rambaut, E.C. Holmes and P.E. Harvey, The epidemic behaviour of the hepatitis C virus, Science 292 (2001), pp. 2323-2325.

Ross et al., 2000 R.S. Ross, S. Viazov, K. Renzing-Köhler and M. Roggendorf, Changes in the epidemiology of hepatitis C infection in Germany: shift in the predominance of hepatitis C subtypes, J Med Virol 60 (2000), pp. 122-125.

Simmonds et al., 2005 P. Simmonds, J. Buck, C. Combet, G. Deleage, N. Enomoto and S. Feinstone et al., Consensus proposal for a unified system of nomenclature of hepatitis C virus genotypes, Hepatology 42 (2005), pp. 962-973.

Verbeeck et al., 2006 J. Verbeeck, P. Maes, P. Lemey, O.G Pybus, E. Wollants and E. Song et al., Investigating the origin and spread of hepatitis C virus genotype 5a, J Virol 50 (2006), pp. 4220-4226. 